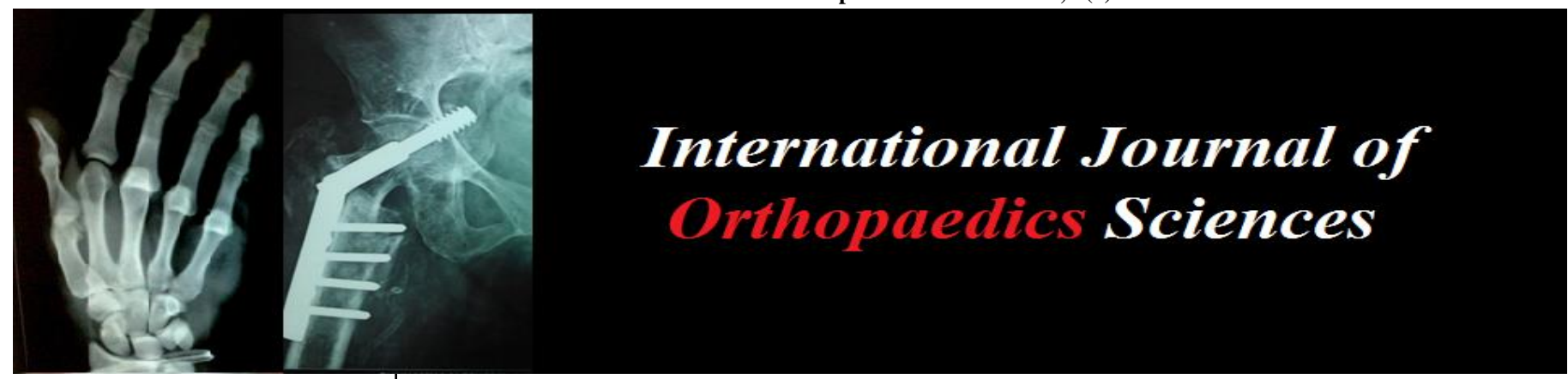

ISSN: $2395-1958$

IJOS 2019; 5(3): 805-809

(C) 2019 IJOS

www.orthopaper.com

Received: 11-05-2019

Accepted: 15-06-2019

Dr. Vinod Kumar Singh Professor, Department of orthopedics, AN Medical College Gaya, Bihar, India
Correspondence

Dr. Vinod Kumar Singh

Professor, Department of

orthopedics, AN Medical College

Gaya, Bihar, India

\section{A comparative study of tension band wiring and cannulated screw fixation for medial malleolar fractures}

\section{Dr. Vinod Kumar Singh}

DOI: https://doi.org/10.22271/ortho.2019.v5.i3n.1630

\section{Abstract}

Background: Ankle fractures have been the subject of numerous studies and articles regarding the mechanism of injury, classification and treatment modalities.

Objective: To compare the clinical, radiological and functional outcome of medial malleolar fracture management by cannulated screw fixation and tension band wiring.

Methods: This is a randomized comparative study done on 40 patients between the age group of 15 to 65 years. The study period was from September 2013 to September 2015. Twenty patients each were treated with cannulated screw fixation and tension band wiring respectively. Outcome measure includes clinical and radiological union. Functional outcome was assessed by using modified ankle score of Olerud and Molander.

Result: Radiological union was seen at 11.6 weeks for cannulated screw fixation and 9.2 weeks for tension band wiring [p value 0.03]. Based on our assessment parameters $80 \%$ had excellent to good outcome $20 \%$ had fair to poor outcome for cannulated screw fixation, where as $90 \%$ had excellent to good outcome and $10 \%$ had fair outcome for tension band wiring [p value 0.04].

Keywords: ankle fracture, medial malleolar fracture, cannulated screw, tension band wiring

\section{Introduction}

Ankle fractures are among the most common injuries treated by orthopaedic surgeons [1, 2]. Injuries around the ankle joint cause destruction of not only the bony architecture, but also often the ligamentous and soft-tissue components ${ }^{[3]}$. With fractures of the ankle, only slight variation from normal is compatible with good joint function. The best results are obtained by anatomical joint restoration; the method used to accomplish this may be either closed manipulation or open reduction and internal fixation. For most fractures, the latter method most often ensures anatomical joint restoration and union.

\section{Classification: Lauge-hansen classification}

A classification system based on causative mechanism of injury was proposed by Ashurst and Bromer in 1922, and expanded by Lauge-Hansen in 1950 following cadaveric investigations [4]. It employs two words and a number. The first word describes the position of the foot at the time of fracture (supination or pronation), the second the deforming force at the ankle (abduction, adduction, internal rotation or external rotation). There are four resulting classes of injury: Supination external rotation (SER), pronation external rotation (PER), supination adduction (SAD), and pronation abduction (PAB). The most common pattern of injury is SER $(60 \%)$ followed by SAD injuries $(20 \%)$ and then those occurring in pronation $(20 \%)$. PAB fractures and PER fractures comprise $8 \%$ and $12 \%$ of ankle fractures respectively. The LaugeHansen classification historically indicated the process of closed manipulation required to reverse displacement and reduce the fracture, but in the era of surgical fixation this classification system remains helpful in directing management.

\section{Treatment}

Medial malleolar fractures occur commonly and are often treated surgically. For any given 
fracture, treatment depends on the fracture configuration, and the clinical scenario. Multiple fixation options exist, including cortical or cancellous screws, tension band wiring, plates and screws, and even suture anchors according to some reports.

\section{Materials and Methods}

This is a hospital based randomized experimental comparative study done in the department of orthopedics, A N Medical College Gaya, India, between the period of Sept, 2013 to Sept, 2014 in 40 patients with medial malleolus fractures attending casualty and OPD service, after due approval from ethical committee of the institute and informed written consent were obtained. The patients were divided into two groups, group 1 and group 2. Group 1 patients received cannulated screw fixation and group two received tension band wiring as treatments. They were assessed with anteroposterior, lateral and mortise views x-rays. Routine investigations were done. Patients with mental and physical inability to cooperate and presence of serious complicating medical conditions and inability to obtained informed written consent for operation were excluded. Data collected were analyzed by using descriptive statics like mean and percentage.

Surgical techniques: All the patients in this study were operated upon under spinal anesthesia. The patient was positioned supine and an Esmarch tourniquet was applied to the mid-thigh. After routine skin preparation and draping, we made an anteromedial incision that began approximately $2 \mathrm{~cm}$ proximal to the fracture line, extended distally and slightly posteriorly, and ended approximately $2 \mathrm{~cm}$ distal to the tip of the medial malleolus. We prefer this incision for two reasons: first, the tibialis posterior tendon and its sheath are less likely to be damaged, and second, the surgeon is able to see the articular surfaces, especially the anteromedial aspect of the joint, which permits accurate alignment of the fracture. Skin was handled with care, and the flap reflected intact with its underlying subcutaneous tissue. The blood supply to the skin of this area is poor, and careful handling is necessary to prevent skin sloughing. We protect the great saphenous vein and its accompanying nerve. A small fold of periosteum commonly is interposed between the fracture surfaces. We removed this fold from the fracture site with a curette or periosteal elevator, exposing the small serrations of the fracture. We debrided small, loose osseous or chondral fragments; large osteochondral fragments were preserved. With a small bone- holding clamp, the displaced malleolus was brought into normal position and, while holding it there, internally fixed with either malleolar screw or tension-band wiring.

\section{Malleolar screw fixation}

In group1 patients a $3.2 \mathrm{~mm}$ hole was drilled while distal fragment was held reduced with a pointed clamp or with two Kirschner wires bent to stay out of way as temporary fixation devices. Length of hole was measured, and a malleolar screw was inserted without tapping. Kirschner wires were removed after screw is tightened.

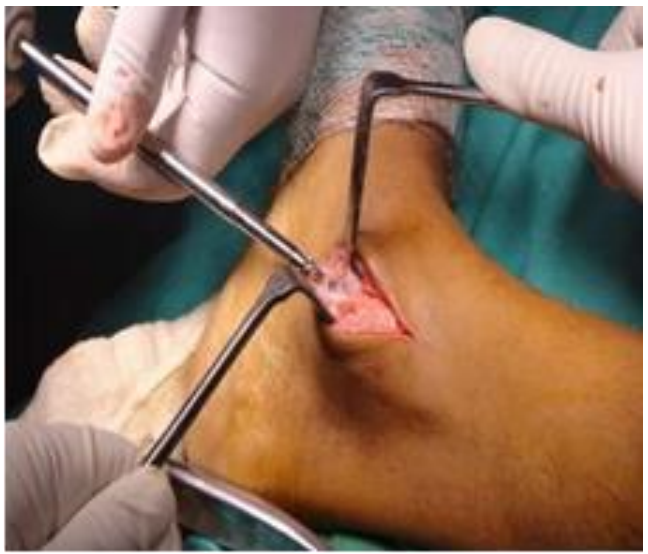

Fig 1: Showing cannulated screw fixation

\section{Tension band wiring}

In group 2 patients the fracture was internally fixed with two 2-mm smooth Kirschner wires drilled perpendicular to the fracture line. The Kirschner wires should be parallel, and their ends were bent at $90^{\circ}$ angles. This will eventually prevent the figure-of-eight wire from slipping over the exposed ends of the Kirschner wires. A stainless steel $1.2 \mathrm{~mm}$ AO wire was passed through the previously drilled hole and around the bent ends of the Kirschner wires in a figure-of-eight configuration. The wire was then tightened. We were carefully inspected the interior of the joint, particularly at the superomedial corner, to make sure that Kirschner wires or the screw had not crossed the articular surfaces. In conditions were image intensifier was available, we made roentgenograms to verify the position of the screw or the Kirschner wires and any faulty insertion could be avoided. Screen control was used in 3 cases only in our study. At the end of operation we deflated the tourniquet, obtained hemostasis, and closed the wound with interrupted suture. We avoided tight stitches to prevent necrosis of the skin edges. We applied thick padding and a posterior plaster splint with the ankle in neutral position.

\section{After treatment}

The ankle is immobilized in a posterior plaster splint with the ankle in neutral position and elevated. Postoperative $\mathrm{x}$-ray was taken with anterior, lateral, and mortise views.

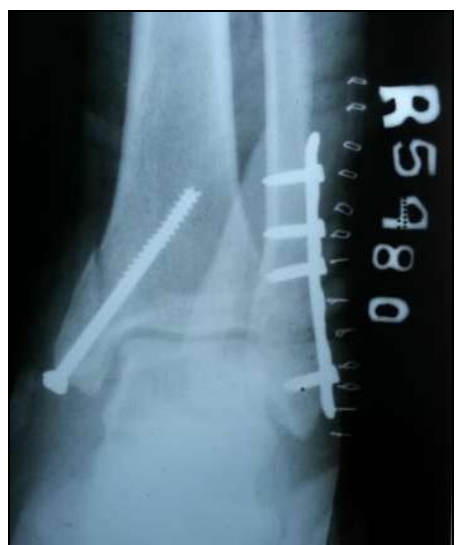

Fig 2: Showing postop x-ray [Group 1]

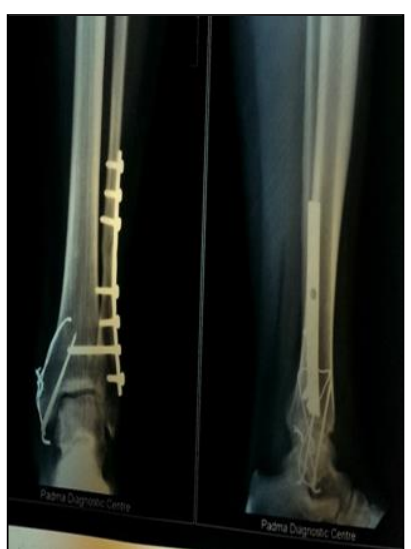

Fig 3: Showing postop x-ray [Group 2] 


\section{Follow up}

All the patients were reviewed at 10 to 14 days, six weeks, three months, six months and one year after operation. At each assessment we perform a physical and radiological examination. After 10 to 14 days the stitches were removed and the wound examined and any complication was reported and treated accordingly. The posterior plaster splint was replaced with a removable splint and range of motion exercises were then begun. Weight-bearing was restricted for 6 weeks after which the splint discarded and partial weight bearing started. Full weight bearing was allowed after 12 weeks.

We evaluated the patients clinically, radiologically, and functionally using a modification of the scoring system proposed by Olerud and Molander ${ }^{[5]}$. The scores for each component of this scale were assessed by the use of a questionnaire, in combination with clinical objective criteria. The scoring scale has a maximum of 100 points $(>91$ excellent results, 81-90 good results, 71-80 fair results, $<70$ poor results).

Table 1: The modified ankle score of olerud and molander

\begin{tabular}{|c|c|}
\hline Parameter & Score \\
\hline \multicolumn{2}{|c|}{ 1. Pain } \\
\hline None & 25 \\
\hline Minor & 20 \\
\hline During sports & 15 \\
\hline During walking & 5 \\
\hline Constant and severe & 0 \\
\hline \multicolumn{2}{|c|}{ 2.Stiffness } \\
\hline None & 10 \\
\hline In the morning & 5 \\
\hline Constant & 0 \\
\hline \multicolumn{2}{|c|}{ 3. Swelling } \\
\hline None & 10 \\
\hline In the evening & 5 \\
\hline Constant & 0 \\
\hline \multicolumn{2}{|c|}{ 4. Stair climbing } \\
\hline Normal & 10 \\
\hline Impaired & 5 \\
\hline Impossible & 0 \\
\hline \multicolumn{2}{|l|}{ 5. Sports } \\
\hline Normal & 10 \\
\hline Impaired & 5 \\
\hline Impossible & 0 \\
\hline \multicolumn{2}{|c|}{ 6. Supports } \\
\hline None & 10 \\
\hline Tape or wrap & 5 \\
\hline Stick or crutch & 0 \\
\hline \multicolumn{2}{|c|}{ 7. Daily activities } \\
\hline Normal & 10 \\
\hline Reduced & 5 \\
\hline Impaired & 0 \\
\hline Total & 100 \\
\hline
\end{tabular}

\section{Results}

The mean duration of operation for cannulated screw fixation was 32.8 minute and tension band wiring was 45.7 minute. Mean age for group 1 was 38.4 years and for group 2 was 37.9 in group $140 \%$ were male and $60 \%$ were female where as in group 2 sex distribution were equal. Mean time for radiological union was 11.6 weeks in group one and in group 2 it was 9.2 weeks. According to the modified ankle scoring system of Olerud and Molander, 2 (20\%) patients in group1 and $4(40 \%)$ patients in group2 were excellent: good in 14 $(70 \%)$ patients in group1 and $14(70 \%)$ in group 2: fair in 2
(20\%) patients in group 1 and $2(20 \%)$ in group 2: poor in 2 (20\%) patients in group 1 and non in group 2 patients. Excellent and good results were achieved in $80 \%$ in group 1 patients and $90 \%$ in group 2 patients. $(\mathrm{p}=0.04)$.

Table 2: Mean time for radiological union

\begin{tabular}{|c|c|c|}
\hline Groups & Mean Time [weeks] & P Value \\
\hline Group 1 [Screw] & 11.6 & \multirow{2}{*}{0.03} \\
\hline Group 2 [TBW] & 9.2 & \\
\hline
\end{tabular}

Table 3: Follow up assessment using modified Olerud and Molander scoring system

\begin{tabular}{|c|c|c|}
\hline Outcome & Group 1 [n=20] & Group 2 [n=20] \\
\hline Excellent & 2 & 4 \\
\hline Good & 14 & 14 \\
\hline Fair & 2 & 2 \\
\hline Poor & 2 & 0 \\
\hline
\end{tabular}

\begin{tabular}{|c|c|c|c|}
\hline Outcome & Group 1 & Group 2 & P value \\
\hline Excellent+Good & $80 \%$ & $90 \%$ & 0.04 \\
\hline
\end{tabular}

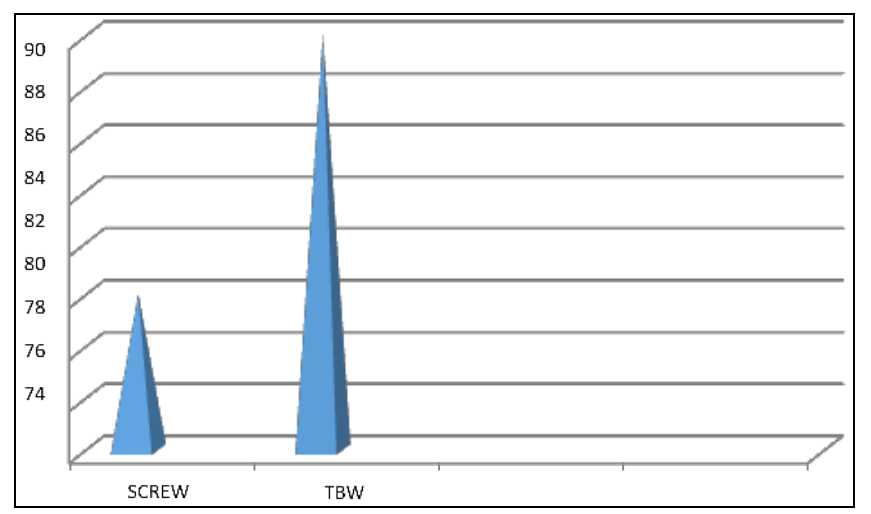

Fig 4: Showing excellent and good results [\%]

\section{Complications \\ Intraoperative}

During reduction of the fracture, use of instruments such as pointed clamps to align and hold the fragments can cause further comminution or crushing of the medial malleolus especially in osteoporotic bone. We developed such complication in one case and it was difficult to fix such fracture using malleolar screws. We removed the bone clamp and fixed the fracture using two kirschner wires and tensionband wire.

\section{Postoperative}

Skin necrosis

Necrosis of the skin edges at the site of the operation was recorded in three patients $(7.5 \%)$ two in group 1 and the other in group 2 patients. They were treated with meticulous debridement of the necrotic skin and dressing.

\section{Wound infection}

It developed in 4 patients (10\%) two in group1 and two in group2. The condition resolved with local wound care, regular dressing and antibiotics.

\section{Delayed union}

Two cases $(5 \%)$ out of 40 cases develops this complication. The patients were female aged 53 years and male aged 40 years, they are treated by malleolar screw (group 1 patients). The patients continue to have pain on the medial side of ankle joint after 13 weeks of fixation and the fracture takes around 
17 weeks to unite radiologically. During this period, we continue in protection of the fracture with splint and full weight-bearing was also delayed.

\section{Limitation of movement.}

Clinically the loaded dorsal range of movement was measured at the final follow-up examination by standing the patients on a small elevated box with the knee and hip flexed. Both ankles were measured. $80 \%$ of group 1 patients compared with $70 \%$ of group 2 patients had ankle dorsiflexion greater than 15 degrees $(p=0.6)$. Osteoarthritis was assessed by radiological observation of the joint space, osteophyte formation and ligament calcification. However, we faced two cases of osteoarthritis in this study, the first was a male aged 38 years (Group 1) and the other was a lady aged 42 years (Group 2).

\section{Complications}

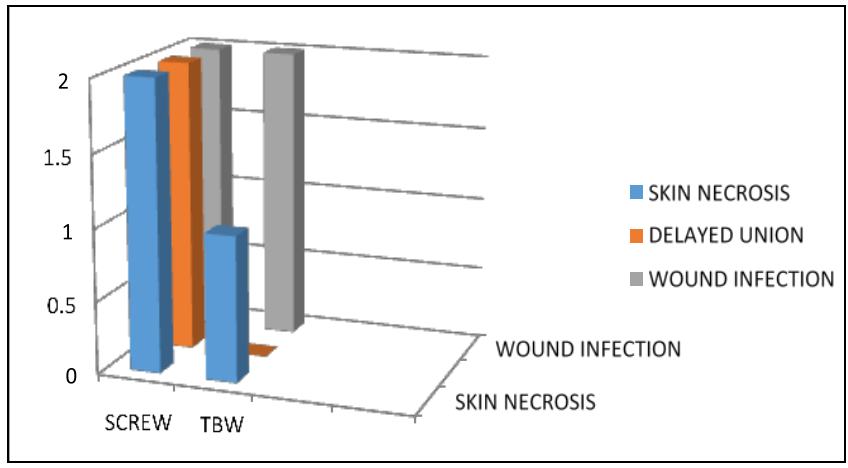

Fig 5: Showing complications

\section{Discussion}

According to the modified ankle scoring system of Olerud and Molander ${ }^{5}$ the current study showed that excellent and good results were achieved in $80 \%$ in group 1 patients (Treated with malleolar screws) and $90 \%$ in group 2 patients (Treated with tension-band wiring) (The difference was significant $\mathrm{p}=0.04)$. This agrees with the results of Sang Hanko and Young Junpark ${ }^{[6]}$ who were achieved excellent and good results in about $78 \%$ of cases treated with malleolar screws and $89 \%$ of cases treated with tension-band wiring.

In this study the mean time for radiological bone union was 11.6 weeks (Ranging from 8 to 18 weeks) for group1 patients and 9.2 weeks (Ranging from 6 to 12 weeks) for group2 patients (Significant difference $\mathrm{p}=0.03$ ). This is similar to Nurul SK and Shahidi $P{ }^{[7]}$ study that was reported a mean time of 12 weeks for malleolar screws and 9 weeks for tension-band wiring.

We had experienced two cases of delayed union (5\%) out of 40 cases of the study and no non-union developed. The patients were female aged 53 years and male aged 40 years treated with malleolar screw fixation and the fracture took around 17 weeks to unite. This result slightly differs from the results of Nurul SK and Shahidi P ${ }^{[7]}$ who achieved $100 \%$ union rate in both groups without any case of delayed union. Ostrum and Litski ${ }^{[8]}$ recently demonstrated the biomechanics advantages of the tension-band over other fixation techniques for medial malleolus. When resisting pronation forces and applying compression force tension-band were four times stronger than malleolar screw. This might explain the faster union rate we were achieved in group2 patients (Mean of 9.2 weeks) as compared with group1 patients (Mean of 11.6 weeks).
Rovinsky et al. ${ }^{[9]}$ in his study showed that the tension-band is more technically advantageous over other types of fixation for fixation of small fragment fracture of medial malleolus and is not recommended for the fixation of vertical fracture. We agree with these results as in our study we fixed few vertical fractures with horizontally directed malleolar screws but we excluded them from the comparison groups.

Screw fixation alone may provide poor stability against torsional forces ${ }^{[10]}$. This may requires an additional point of fixation, which may be a second screw or a Kirschner wire. In the current study we use additional point of fixation (second screw and $\mathrm{K}$. wire) in two cases in which the fragment was large and tend to rotate.

Limitation of movements and swelling of the ankle are usually the result of neglect in treatment of soft tissue. Although better range of motion was noticed in group1 patients $(80 \%)$ as compared with group $2(70 \%)$, it did not reach significance $(\mathrm{p}=0.6)$. This could be attributed to wide soft tissue dissection that was needed with the use of tensionband. These results may show similarity with the results of Nurul SK and Shahidi $\mathrm{P}^{[7]}$ who reported in his study that the group treated with malleolar screw showed better range of motion.

\section{Conclusion}

Our study showed that tension-band wiring may be more valid option for internal fixation of medial malleolar fractures. The tension-band wiring is more technically advantageous for small fragment fixation of medial malleolar fractures. The tension-band wiring may be more available and its usage could translate into overall cost saving when applied to the large number of ankle fractures treated surgically in our country. Screw fixation alone may provide poor stability against torsional forces and may require an additional point of fixation which may be a second screw or a Kirschner wire. The faster radiological union which was achieved with the use of tension-band wiring as compared with malleolar screw could be added to the overall advantages of using this technique. During surgical fixation of medial malleolar fractures excessive pressure with bone clamps to hold the fracture reduction should be avoided to prevent crushing of the fragment particularly if the bone is osteoporotic, the fracture reduction instead can hold temporarily with $\mathrm{k}$ wires. Careful and meticulous soft tissue handling is important for prevention of postoperative wound complications, delayed union and joint stiffness.

\section{Summary}

Medial malleolar fractures occur commonly and are often treated surgically. Our study was designed to compare clinical, radiological, functional outcome of medial malleolar fracture of tibia treated by cannulated screw fixation and tension band wiring.

The study was conducted in the department of orthopedics, A $\mathrm{N}$ Medical College Gaya, India, for a period of two years from September 2016 to September 2018. This is a comparative study in which 40 consecutive cases of medial malleolar fracture of tibia fitting in the inclusion criteria, were subjected to open reduction and internal fixation by using cannulated screw in 20 patients and tension band wiring in 20 patients

Our current study shows that tension band wiring is better option for treatment of medial malleolar fractures. It is easily available and cost effective and provides a good and stable fixation in comparison with malleolar screw fixation. Time 
taken for radiological union is lesser in patients treated with tension band wiring. In our study the mean time for radiological bone union was 11.6 weeks for group 1 (screw) patients and

9.2 weeks for group 2 (TBW) patients (significant difference $\mathrm{p}=0.03$ ). According to the modified ankle scoring system of Olerud and Molander [5] the current study showed that excellent and good results were achieved in $80 \%$ in group 1 patients (treated with malleolar screws) and $90 \%$ in group 2 patients (treated with tension-band wiring) (the difference was significant $\mathrm{p}=0.04)$. In our study there was no significant difference between two groups in term of range of motion.

The preliminary results of the current study suggest that tension-band wiring may be more valid option for internal fixation of medial malleolar fractures. Screw fixation alone may provide poor stability against torsional forces and may require an additional point of fixation. The faster radiological union and better functional results were achieved with the use of tension-band wiring as compared with malleolar screw.

\section{References}

1. Kannus P, Parkkari J, Niemi S et al. Epidemiology of osteoporotic ankle fractures in elderly persons in Finland. Ann Intern Med. 1996; 125(12):975-8.

2. Court-Brown CM, McBirnie J, Wilson G. Adult ankle fractures: an increasing problem. Acta Orthop Scand. 1998; 69:43-7.

3. Hughes JL Weber, Willenger H, Kuner EH. Evaluation of ankle fractures; non-operative and operative treatment. Clin Orthop Relat Res. 1979; 138:111-19.

4. Lauge Hansen N. Fractures of the ankle. Combined experimental-surgical and experimental roentgenologic investigations. Arch Surg. 1950; 60(5):957-85.

5. Olerud C, Molander H. A scoring scale for symptom evaluation after ankle fracture. Arch Orthop Trauma Surg. 1984; 103:190-4.

6. Sang-Hanko M, Young-Jun Park D. Comparison between screw fixation and tension-band wiring for medial malleolus fractures. Korean Society of Foot Surgery. 2002; 6(1):41-4.

7. Nurul SK, Shahidi P. Comparative study of malleolar fractures by tension-band and malleolar screw. J Orthod 1998; 12(1):13-9.

8. Ostrum RF, Listsky AS. Tension-band fixation of medial malleolar fracture. J Orthop Trauma. 1992; 6(4):464-8.

9. Rovinsky D, Haskell A, Liu Q, Paiement GD, Robinovitch S. Evaluation of a new method of small fragment fixation in a medial malleolus fracture model. J Orthop Trauma. 2000; 14(6):420-5.

10. Savage T, McGarry J, Stone PA. The internal fixation of ankle fracture. Clin Podiat Med Surg 1. 